\title{
Does esophageal pressure monitoring reliably permit to estimate transpulmonary pressure in children?
}

\author{
Guillaume Mortamet $^{1-4 *}$, Nicolas Nardi ${ }^{1,2}$, Nancy Poirier ${ }^{2,5}$, Sandrine Essouri ${ }^{2,6}$, Brigitte Fauroux ${ }^{2-4,7}$, Philippe Jouvet ${ }^{1,2}$ and Guillaume \\ Emeriaud $^{1,2}$ \\ ${ }^{1}$ Pediatric Intensive Care Unit, CHU Sainte-Justine, Montreal (QC), Canada \\ ${ }^{2}$ Université de Montréal, Montréal (QC), Canada \\ ${ }^{3}$ Université de Paris-Est, Créteil, France \\ ${ }^{4}$ Unité INSERM U955, Créteil, France \\ ${ }^{5}$ Department of Cardiac Surgery, CHU Sainte-Justine, Montreal (QC), Canada \\ ${ }^{6}$ Department of Pediatrics, CHU Sainte-Justine, Montreal (QC), Canada \\ ${ }^{7}$ Pediatric Noninvasive Ventilation and Sleep Unit, Hospital Necker, Paris, France
}

\begin{abstract}
Objectives: This study aims to determine whether Transpulmonary Pressure can be measured in children using Esophageal Pressure as a surrogate of Pleural Pressure $\left(\mathrm{P}_{\mathrm{PL}}\right)$. To reach this goal, we wanted to validate the reliability of two Esophageal Pressure recording methods compared to direct $\mathrm{P}_{\mathrm{PL}}$ measurement in situ.

Study design: This is a prospective study.

Methodology: Mechanically ventilated children were included if they had at least one chest tube. $\mathrm{P}_{\mathrm{PL}}$ was directly measured into the existing chest tube $\left(\mathrm{P}_{\mathrm{CH}-\mathrm{TUBE}}\right)$. Esophageal Pressure was measured by two methods: a catheter mounted pressure transducer system $\left(\mathrm{P}_{\text {ES-REF }}\right)$ and the preexisting nasogastric feeding tube pulled out in order to be located in the mid third of the esophagus $\left(\mathrm{P}_{\mathrm{ES}-\mathrm{FT}}\right)$.

Results: Twelve patients were enrolled, and eight patients (median age: 4 months) were included in the analysis. For each method, the 2 measurements obtained with the same method were concordant. In the Bland-Altman analysis, the limits of agreement were wide for all between-method comparisons, from \pm 8 to $\pm 15 \mathrm{~cm} \mathrm{H}_{2} \mathrm{O}$.

Conclusion: Prior to consider its use in clinical practice, in particular for the titration of the ventilatory support, it is essential to conduct more research in order to validate the measurement technique of Esophageal Pressure and confirm that it can accurately reflect the $\mathrm{P}_{\mathrm{PL}}$.
\end{abstract}

\section{Introduction}

The measurement of Transpulmonary Pressure $\left(\mathrm{P}_{\mathrm{TP}}\right)$, i.e. the difference between alveolar pressure and pleural pressure $\left(\mathrm{P}_{\mathrm{PL}}\right)$, is the best conceptual step to monitor the effective pressure transmitted to the lung itself [1]. Because $\mathrm{P}_{\mathrm{PL}}$ is difficult to measure directly in the clinical setting, several physiological studies suggested that Esophageal Pressure $\left(\mathrm{P}_{\mathrm{ES}}\right)$ could be used as a surrogate of $\mathrm{P}_{\mathrm{PL}}$ in adults, while airway pressure is measured in the ventilator circuit [2-5].

More recently, a few studies performed in adults demonstrated a potential benefit of $\mathrm{P}_{\mathrm{ES}}$ monitoring in ventilator management. This measurement has been shown to be helpful in optimizing patientventilator interaction [6], for the titration of Positive End-Expiratory Pressure (PEEP) [7], and in the weaning management [8]. In children, although the literature in this field is scarce, the recent international consensus conference on pediatric acute respiratory distress syndrome PALICC [9] stresses that $P_{E S}$ measurement could be useful, but there is no evidence regarding the method used to measure and interpret this value.

Despite data demonstrating usefulness of $\mathrm{P}_{\mathrm{ES}}$ measurement in critically ill patients, several confounding factors that can affect the accuracy of $\mathrm{P}_{E S}$ have been highlighted in the literature [10-13]. To compensate for these factors, some authors apply a correction factor to estimate $\mathrm{P}_{\mathrm{PL}}[10,12,14]$, but the use and the level of the correction factor is still controversial $[15,16]$. In addition, due to the pediatric population heterogeneity in terms of age, weight, and conditions modifying the chest wall activity, we hypothesize that $\mathrm{P}_{\mathrm{ES}}$ varies importantly from a child to another. In this specific population, the way to measure $\mathrm{P}_{\mathrm{ES}}$ as a tool to estimate $\mathrm{P}_{\mathrm{PL}}$ needs first to be validated before exploring its potential utility in future clinical studies. This study aims to determine whether $\mathrm{P}_{T \mathrm{P}}$ can be measured in children using $\mathrm{P}_{\mathrm{ES}}$ as a surrogate of $\mathrm{P}_{\mathrm{PL}}$. To reach this goal, we wanted to validate the reliability of two $\mathrm{P}_{\mathrm{ES}}$ recording methods compared to direct $\mathrm{P}_{\mathrm{PL}}$ measurement in situ.

\section{Methods}

This is a prospective study, conducted in the Pediatric Intensive Care Unit (PICU) of CHU Sainte-Justine, a university affiliated

*Correspondence to: Guillaume Mortamet, Pediatric Intensive Care Unit, CHU Sainte-Justine, 3175 Côte Sainte-Catherine, Montreal, Québec, Canada, E-mail: gmortamet@chu-grenoble.fr

Key words: mechanical ventilation, pleural pressure, esophageal pressure, work of breathing, pediatric intensive care unit

Received: September 06, 2018; Accepted: September 25, 2018; Published: September 28, 2018 
pediatric hospital, from November 2016 to September 2017. The study protocol was approved by the Ethics Committee of CHU Sainte-Justine and written informed consent was obtained from the parents or legal tutor.

\section{Patients}

Consecutive children aged between 7 days and 18 -years old admitted to the PICU, intubated and mechanically ventilated were eligible in the study. The screening was performed by a research assistant every working day. Eligible patients were included if they required invasive ventilation for more than 4 hours according to the prescription of the attending physician and they had at least one chest tube. Patients were excluded if they had one of the following criteria: (i) contraindications to the placement of a new nasogastric tube, (ii) hemodynamic instability, (iii) severe respiratory instability, (iv) persistent pleural effusion or pneumothorax despite the chest-tube, (v) bronchopleural fistula, (vi) recent thoracic hemorrhage, (vii) delayed sternal closure at the time of study, (viii) Significant pericardial effusion and (ix) if a limitation of life support treatments was discussed or decided. Detailed inclusion and exclusion criteria are given in Supplementary Material.

\section{Protocol and data analysis}

After verification of the permeability of the chest tube and absence of active air leak or pleural effusion, Pleural Pressure in the chest tube $\left(\mathrm{P}_{\mathrm{CH}-\mathrm{TUBE}}\right)$ was directly measured by a pressure transducer connected through a needle inserted into the existing chest tube, as close as possible to the patient, and the tube was occluded distally to the needle. The position of the chest tubes was not controlled for, and they had multiple lateral holes. The tube was flushed by $0.5-1 \mathrm{ml}$ of air in order to eliminate potential secretions (patency of the column of air) before the measurements, which were obtained after pressure equilibrium.

Esophageal Pressure was measured by two methods. The reference esophageal pressure $\left(\mathrm{P}_{\mathrm{ES}-\mathrm{REF}}\right)$ was measured using a $2.1 \mathrm{~mm}$ external diameter catheter mounted pressure transducer system with two integrated pressure transducers, mounted 5 and $25 \mathrm{~cm}$ from the distal tip (Gaeltec, Dunvegan, Isle of Skye, the UK) inserted nasally. The placement of the $\mathrm{P}_{\mathrm{ES}}$ transducer was first estimated with the distance from the bridge of the patient's nose, to the ear lobe and down to the xiphisternum. The appropriate position was checked by the presence of (i) cardiac oscillations on the $\mathrm{P}_{\mathrm{ES}}$ trace, (ii) a positive $\mathrm{P}_{\mathrm{GAS}}$ value during a gentle pressure on the abdomen and (iii) a negative deflection during inspiration in spontaneous breathing patients. The occlusion test during a chest compression, as suggested by Baydur et al. was not performed in case of recent cardiac surgery [17].

The second esophageal pressure method tested was based on the feeding tube $\left(\mathrm{P}_{\mathrm{ES}-\mathrm{FT}}\right)$. After the removal of the Gaeltec probe, the preexisting nasogastric feeding tube was pulled out in order to be located in the mid third of the esophagus, according to the feeding tube position checked on the last available chest X-ray. The nasogastric tube was connected to a pressure transducer and gently flushed with $5 \mathrm{ml}$ of air before the pressure measurement. After measurements, the nasogastric tube was repositioned in gastric position.

All the signals were simultaneously recorded using an acquisition system (NeuroVent Monitor XIII), run on a PC computer and displayed and analyzed using a specific software (NeuroVent Research, Toronto, Canada). $\mathrm{P}_{\text {CH-TUBE}}, \mathrm{P}_{\mathrm{ES}-\mathrm{REF}}$, and $\mathrm{P}_{\mathrm{ES} \text {-FT }}$ were calculated as the average of the end-expiratory values of all consecutive breaths observed during two stable periods of 10 seconds each (exempt of intervention or artifacts).
The patients were supine, with the head of bed elevated to 30 degrees, as a routine matter, and sedated according to the attending physician instructions. During the entire study, "usual" modifications of settings (e.g. adaptation of assist level, changes in $\mathrm{FiO}_{2}$ or Positive End Expiratory Pressure, etc.) considered by the clinical team were permitted and recorded.

Demographic data and patient characteristics, including age, gender, weight, time of measurements, admission diagnostic and comorbidities, Pediatric Risk of Mortality (PRISM) and Pediatric Logistic Organ Dysfunction (PELOD) scores were collected.

\section{Statistical analysis}

Data were expressed as median values (with interquartiles, IQR) for continuous variables, and number and/or frequency (\%) for categorical data. Pearson's determination coefficient $\left(\mathrm{R}^{2}\right)$ was used to evaluate the relationship between the values measured during the two periods with a given method. A correlation was considered as poor, moderate, good and excellent if $\mathrm{R}^{2}$ was lower than 0.5 , between 0.5 and 0.75 , between 0.75 and 0.9 and greater than 0.9 , respectively [18]. Comparisons between $\mathrm{P}_{\mathrm{CH}-\mathrm{TUBE}}$ and $\mathrm{P}_{\mathrm{ES}-\mathrm{REF}}, \mathrm{P}_{\mathrm{CH}-\mathrm{TUBE}}$ and $\mathrm{P}_{\mathrm{ES}-\mathrm{FT} \text {, }}$ and $\mathrm{P}_{\mathrm{ES} \text { - }}$ FTL and $\mathrm{P}_{\mathrm{ES}-\mathrm{REF}}$ followed the method proposed by Bland and Altman, with the calculation of the mean difference and its agreement limit of 95\% [19]. All p-values are two-tailed and considered significant if $p<$ 0.05. Statistical analyses were performed using SPSS 24.0 (SPSS, Inc, Chicago, IL).

\section{Results}

\section{Study population}

Twelve patients reached inclusion criteria and were enrolled. Four patients were posteriori excluded because of technical problems preventing the analysis of the recordings. Exploitable signals were fully available in 8 patients, who were included in the analysis. Median age of patients included was 4 (IQR: $1-4)$ months and 5 were males. They were studied 2 (1-3) days after PICU admission. The patient characteristics are presented in table 1 . The patients were relatively deeply sedated or paralyzed at the time of the study, and spontaneous breathing activity was usually not observed on $\mathrm{P}_{\mathrm{ES}}$ or $\mathrm{P}_{\mathrm{CH}-\mathrm{TUBE}}$ tracings.

\section{Esophageal and pleural pressures analysis}

An example of recording is illustrated in figure 1. Median $\mathrm{P}_{\text {ES-REF }}$ and $\mathrm{P}_{\text {ES-FT }}$ were 3.6 (2.1-7.0) and $8.2(5.3-9.7) \mathrm{cm} \mathrm{H}_{2} \mathrm{O}$, respectively. The median $\mathrm{P}_{\mathrm{CH} \text {-TUBE }}$ was $8.1(2.1-9.7) \mathrm{cm} \mathrm{H}_{2} \mathrm{O}$. For each method, the two measurements conducted with the same method were concordant. The determination coefficient was $0.64,0.95$, and 0.88 for $\mathrm{P}_{\mathrm{CH}-\mathrm{TUBE}}, \mathrm{P}_{\mathrm{ES}}$ and $\mathrm{P}_{\mathrm{ES}-\mathrm{FT}}$, respectively (Figure 2).

We analyzed between-method agreement using Bland-Altman plots (Figure 3). The mean difference (bias) was $0.0 \mathrm{~cm} \mathrm{H}_{2} \mathrm{O}$ between $\mathrm{P}_{\text {CH-TUBE }}$ and $\mathrm{P}_{\text {ES-REF }},-1.0 \mathrm{~cm} \mathrm{H}_{2} \mathrm{O}$ between $\mathrm{P}_{\text {CH-TUBE }}$ and $\mathrm{P}_{\text {ES-FT }}$, and - 2.4 $\mathrm{cm} \mathrm{H} \mathrm{H}_{2} \mathrm{O}$ between $\mathrm{P}_{\text {ES-FT }}$ and $\mathrm{P}_{\text {ES-REF }}$. The limits of agreement were wide: (-8.5-8.6) $\mathrm{cm} \mathrm{H}_{2} \mathrm{O}$ between $\mathrm{P}_{\text {CH-TUBE }}$ and $\mathrm{P}_{\text {ES-REF, }}$ (-15.7-13.8) $\mathrm{cm} \mathrm{H}_{2} \mathrm{O}$ between $\mathrm{P}_{\text {CH-TUBE }}$ and $\mathrm{P}_{\mathrm{ES}-\mathrm{FT}}$, and (-12.8-8.1) $\mathrm{cm} \mathrm{H}_{2} \mathrm{O}$ between $\mathrm{P}_{\mathrm{ES}-\mathrm{FT}}$ and $\mathrm{P}_{\mathrm{ES}-\mathrm{REF}}$

\section{Discussion}

In this study, the observed differences between $\mathrm{P}_{\mathrm{ES}}$ and $\mathrm{P}_{\text {CH-TUBE }}$ are much greater than the value considered as clinically reasonable, especially to guide mechanical ventilation and PEEP titration. Indeed, a cutoff error value $<2 \mathrm{~cm} \mathrm{H}_{2} \mathrm{O}$ was a priori defined as acceptable to 


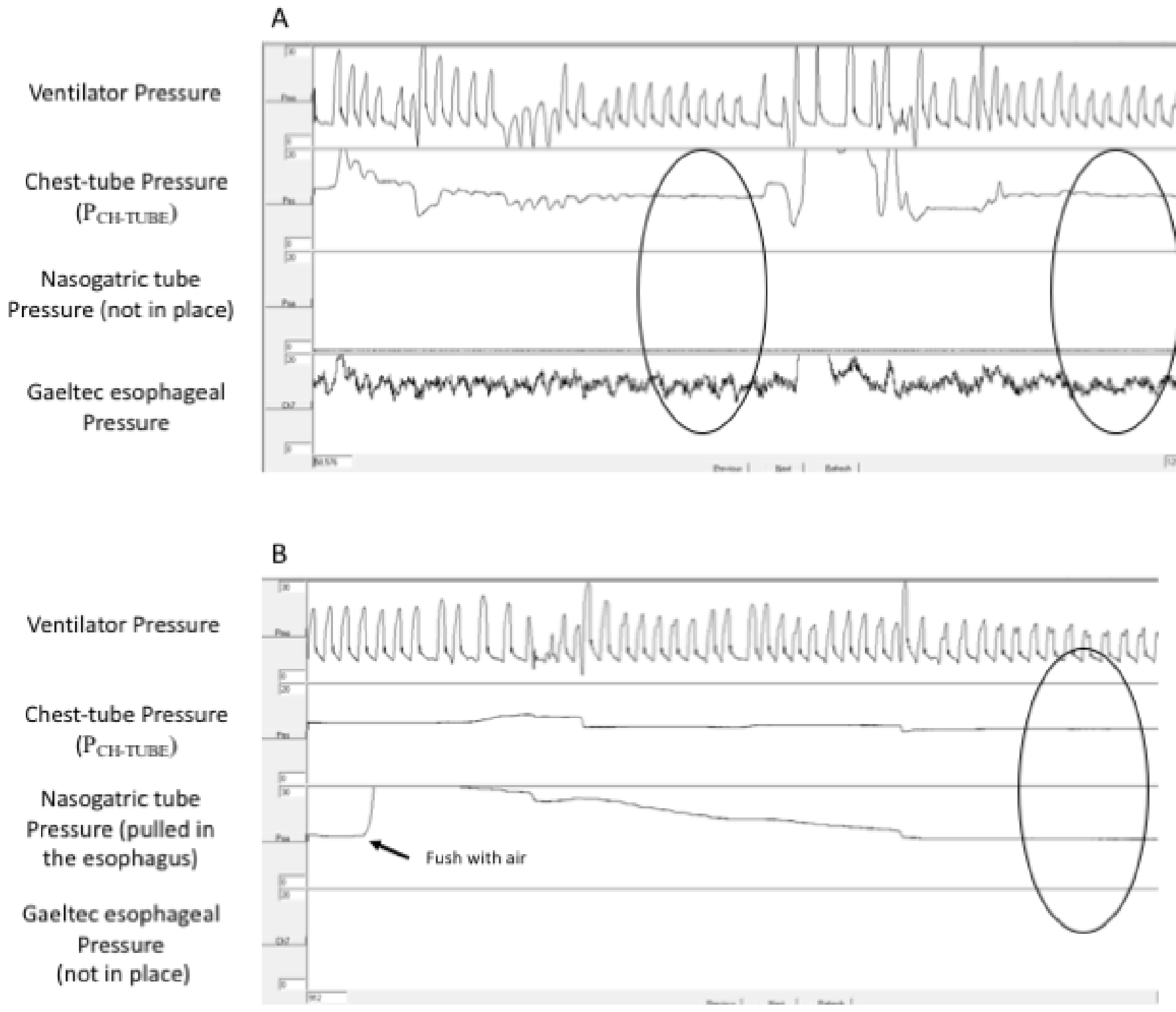

Figure 1. Tracing of a patient showing comparison between $\mathrm{P}_{\text {CH-TUBE }}$ and $\mathrm{P}_{\mathrm{ES}-\mathrm{REF}}$ (step 1, panel A) and between $\mathrm{P}_{\mathrm{CH}-\mathrm{TUBE}}$ and $\mathrm{P}_{\mathrm{ES}-\mathrm{FT}}$ (step 2, panel B). Pressures were calculated as the average of the end-expiratory values of all consecutive breaths observed during two stable periods of 10 seconds each (circles)

A

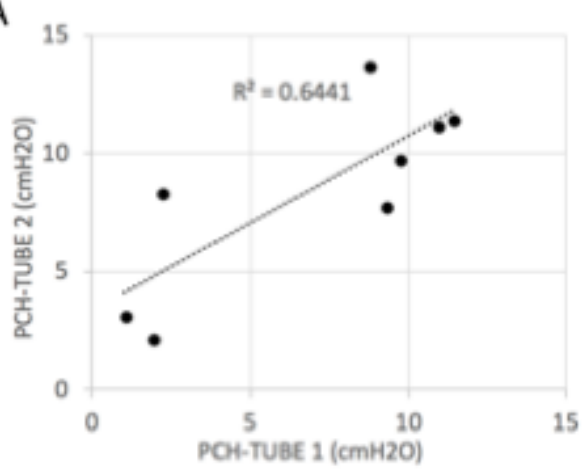

B

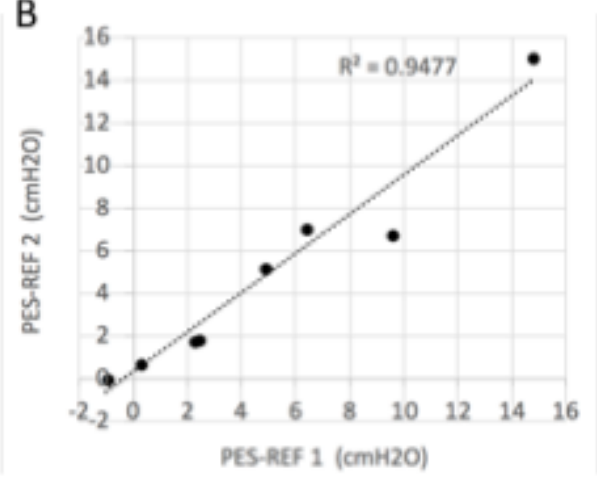

C

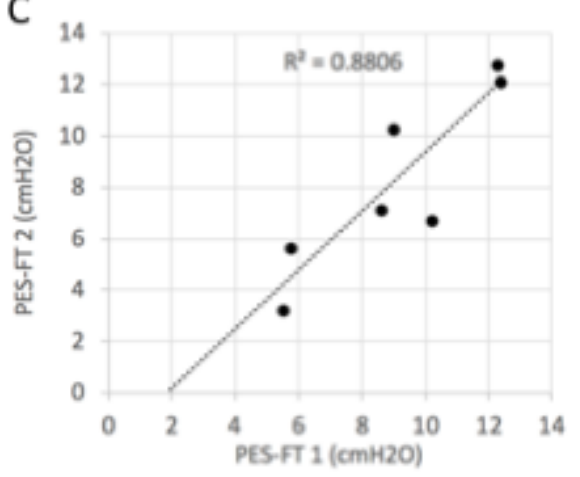

Figure 2. Relationships between the two consecutive measurements performed for each method: $\mathrm{P}_{\mathrm{CH}-\mathrm{TUBE}}$ (panel A), $\mathrm{P}_{\mathrm{ES}-\mathrm{REF}}\left(\right.$ panel B) and $\mathrm{P}_{\mathrm{ES}-\mathrm{FT}}$ (panel C); the first measurement is on the vertical axis and the second on the horizontal axis 
A

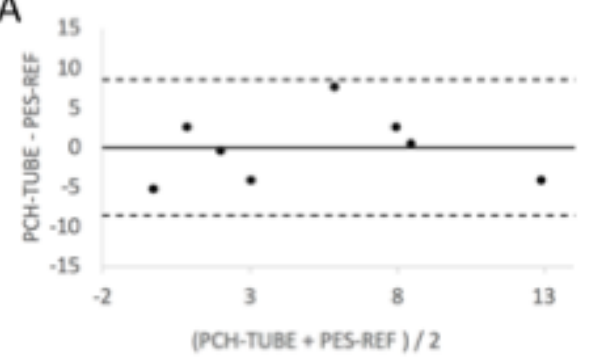

B

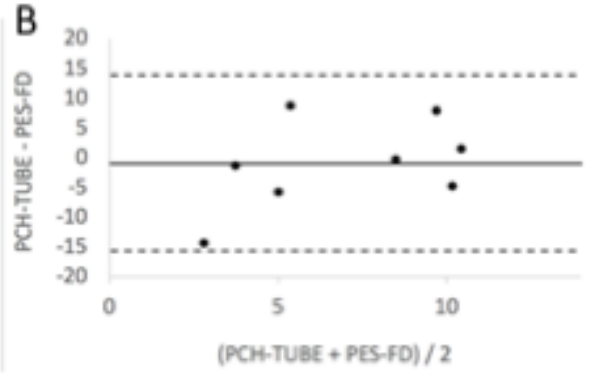

C

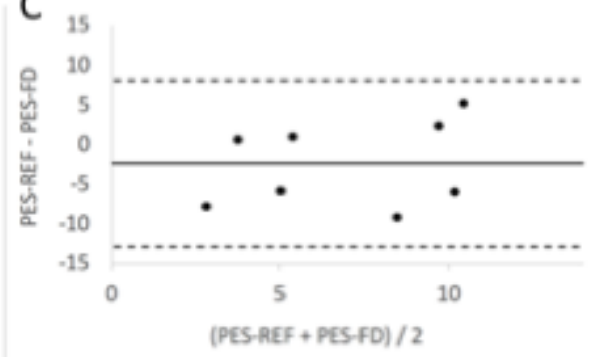

Figure 3. Bland-Altman plots to assess concordance between two different methods of measurement: between $\mathrm{P}_{\text {CH-TUBE }}$ and $\mathrm{P}_{\mathrm{ES}-\mathrm{REF}}$ (panel A), between $\mathrm{P}_{\text {CH-TUBE }}$ and $\mathrm{P}_{\mathrm{ES}-\mathrm{FT}}$ (panel B) and between $\mathrm{P}_{\mathrm{ES}-\mathrm{FT}}$ and $\mathrm{P}_{\mathrm{ES}-\mathrm{REF}}($ panel C)

Table 1. Characteristics of the population $(\mathrm{n}=8)$

\begin{tabular}{|c|c|}
\hline & $\begin{array}{l}\text { Total } \\
\mathbf{n}=\mathbf{8}\end{array}$ \\
\hline Age $(\mathrm{m})$ & 4 (IQR 1-4) \\
\hline Weight (kg) & $5.1(4.6-5.4)$ \\
\hline Male, $\mathrm{n}(\%)$ & $5(63)$ \\
\hline Days between admission and inclusion & $2(1-3)$ \\
\hline Days between MV initiation and inclusion & $2(1-3)$ \\
\hline \multicolumn{2}{|l|}{ Main reasons for PICU admission, $n$} \\
\hline Cardiac postoperative admission & 7 \\
\hline Respiratory failure & 1 \\
\hline \multicolumn{2}{|l|}{ Chronic condition } \\
\hline Congenital cardiac disease, $\mathrm{n}(\%)$ & $7(83)$ \\
\hline \multicolumn{2}{|l|}{ Clinical status } \\
\hline PIM-2 score & $7.5(4.4-7.5)$ \\
\hline PELOD score & $11(2-14)$ \\
\hline $\mathrm{pH}$ & $7.39(7.35-7.44)$ \\
\hline $\mathrm{PaCO}_{2}, \mathrm{mmHg}$ & $44.3(36.8-48.0)$ \\
\hline $\mathrm{HCO}_{3,-}, \mathrm{mmHg}$ & $25.6(23.5-27.7)$ \\
\hline Lactates, $\mathrm{mmol} / \mathrm{L}$ & $1.2(1.1-1.6)$ \\
\hline Hemoglobin, $\mathrm{g} / \mathrm{L}$ & $12.4(10.7-14.0)$ \\
\hline \multicolumn{2}{|l|}{ Hemodynamic status } \\
\hline Pulse, $\min ^{-1}$ & $141(120-152)$ \\
\hline Mean Arterial Pressure, $\mathrm{mmHg}$ & $63(57-69)$ \\
\hline Vasoactive drugs, $\mathrm{n}(\%)$ & $2(25 \%)$ \\
\hline \multicolumn{2}{|l|}{ Ventilator modes and settings } \\
\hline $\begin{array}{l}\text { PRVC, n (\%) } \\
\text { VC, n (\%) } \\
\text { NAVA, n (\%) } \\
\text { PSV, n (\%) }\end{array}$ & $\begin{array}{l}2(25) \\
3(38) \\
1(13) \\
2(25)\end{array}$ \\
\hline Positive End-expiratory Pressure, $\mathrm{cmH}_{2} \mathrm{O}$ & $5(5-6)$ \\
\hline $\mathrm{FiO}_{2}$ & $0.40(0.35-0.51)$ \\
\hline \multicolumn{2}{|l|}{ Respiratory status } \\
\hline $\mathrm{SpO}_{2}, \%$ & $100(98-100)$ \\
\hline Set RR, $\min ^{-1}$ & $30(25-35)$ \\
\hline $\mathrm{V}_{\mathrm{T}}, \mathrm{ml} / \mathrm{kg}$ & $6.8(6.3-7.0)$ \\
\hline \multicolumn{2}{|l|}{ Outcome } \\
\hline Duration of mechanical ventilation, $\mathrm{d}$ & $4(1-5)$ \\
\hline Length of stay in PICU, $d$ & $5(3-11)$ \\
\hline
\end{tabular}

set PEEP, by a panel of pediatric intensive care physicians during the preparation of this study. In our cohort, the difference between $\mathrm{P}_{\text {CH-TUBE }}$ and $\mathrm{P}_{\mathrm{ES}-\mathrm{REF}}$ was $>2 \mathrm{~cm} \mathrm{H}_{2} \mathrm{O}$ in 6 of 8 patients. We therefore failed to confirm that $\mathrm{P}_{\mathrm{PL}}$ could be reliably estimated based on the $\mathrm{P}_{\mathrm{ES}}$. While $\mathrm{P}_{\mathrm{ES}}$ measurement is classically based on a specific esophageal probe or balloon, we also tested if a simpler and less invasive method could provide results as accurate as with the Gaeltec probe. As most critically ill children have a nasogastric tube, we attempted to use this device for $\mathrm{P}_{\mathrm{ES}}$ measurement after appropriate positioning in the mid third esophagus. Again, we failed to show that this simpler method was a reliable estimate of $\mathrm{P}_{\mathrm{ES} \text {-REF }}$.

For decades, $\mathrm{P}_{\mathrm{ES}}$ has been considered a good surrogate of $\mathrm{P}_{\mathrm{PL}}$. This assumption is based on the notion that pressure in the adjacent pleura is transmitted to the esophagus [20,21]. Importantly, $\mathrm{P}_{\mathrm{ES}}$ measurement is one of the reference methods to assess the work of breathing during spontaneous or assisted ventilation. $\mathrm{P}_{\mathrm{ES}}$ swings and the area under the $\mathrm{P}_{\mathrm{ES}}$ curve during inspiration (i.e. Esophageal Pressure-Time Product) accurately provide a very good reflection of the amount of respiratory muscle work, with several relevant clinical applications in children $[22,23]$. Those $\mathrm{P}_{\mathrm{ES}}$-derived data are not based on the absolute $\mathrm{P}_{\mathrm{ES}}$ values, but rather on the relative variations. However, the accuracy of the estimation of $\mathrm{P}_{\mathrm{PL}}$ by $\mathrm{P}_{\mathrm{ES}}$ when absolute values are used (e.g. for titration of the ventilatory support) is much more complex and questionable, in particular because the zeroing of the method is not simple. Despite $\mathrm{P}_{\mathrm{ES}}$ changes have been shown to be similar to $\mathrm{P}_{\mathrm{PL}}$ changes, absolute values of $\mathrm{P}_{\mathrm{ES}}$ tend in general to be less negative than $\mathrm{P}_{\mathrm{PL}}$ both in adults [5] and preterm babies [24]. Several confounding factors could affect the accuracy of $\mathrm{P}_{\mathrm{ES}}$ measurement. The position of the balloon in the esophagus [3], the presence of asymmetrical lung disease [4], the amount of air or liquid to inflate the balloon and its compliance [20] or the posture of the patient [25] are factors well-known to impact the estimation of $\mathrm{P}_{\mathrm{PL}}$ by $\mathrm{P}_{\mathrm{ES}}$. Indeed, the pressure vector generated by the weight of the mediastinal structures has a significant influence on $\mathrm{P}_{\mathrm{ES}}[12,13]$. To compensate for these artifacts, some authors suggest a correction factor that should be applied to interpret $\mathrm{P}_{\mathrm{ES}}$ measurement, but the use and the level of the correction factor is still controversial $[12,16,26]$. Regardless of uncertainties about the interpretation of $\mathrm{P}_{\mathrm{ES}}$, all these artifacts have been considered to be within a clinically acceptable range in adults and $\mathrm{P}_{\text {TP }}$ measurement is now advocated by some experts to identify the optimal ventilator settings in clinical practice [7,27]. Indeed, $\mathrm{P}_{\text {TP }}$, obtained from absolute values of $\mathrm{P}_{\mathrm{ES}}$, is the pressure variable that is the most closely correlated with lung strain and the risk of ventilation induced lung injury [28]. The monitoring of $\mathrm{P}_{\mathrm{TP}}$ is therefore attractive to manage mechanical ventilation. As suggested in the study by Talmor et al., oxygenation and compliance were improved when PEEP titration was guided by $\mathrm{P}_{\mathrm{TP}}$ in patients with Acute Respiratory Distress Syndrome [7]. Although $\mathrm{P}_{\mathrm{ES}}$ and $\mathrm{P}_{\mathrm{TP}}$ could have a greater potential interest to guide mechanical ventilation in children given the heterogeneity of the pediatric population, the literature is scarce in this field [29]. We could legitimately hypothesize that some of the confounding factors described in adults could have a great importance in the pediatric population given their anatomical and physiological specificities. The between-method differences were huge in our study, and clearly beyond an acceptable error when titrating the ventilation. For example, pediatric intensivists would not accept an error of $>2 \mathrm{~cm} \mathrm{H}_{2} \mathrm{O}$ when adjusting the level of PEEP. A 
systematic bias was not the main issue, but rather the wide limits of agreement. A compensation by a given corrective factor, as sometimes done in adults, would therefore not help.

Of course, our study may be limited by important technical issues, although our results highlight that the monitoring of absolute values of $\mathrm{P}_{\mathrm{ES}}$ as a surrogate of $\mathrm{P}_{\mathrm{PL}}$ is greatly complex in children. The direct measurement of $\mathrm{P}_{\mathrm{PL}}$ in situ is technically difficult, although previous data have been reported in adults [30,31] and newborns [24,29]. The site of measurement in the pleural space is important. Yoshida et al. recently demonstrated in an animal model and in human cadavers that the pleural pressure difference between dependent and non-dependent regions could reach 8-10 $\mathrm{cmH}_{2} \mathrm{O}$ [32]. Important discrepancies between $\mathrm{P}_{\mathrm{ES}}$ and $\mathrm{P}_{\mathrm{PL}}$ were also observed in this study, depending on the transducer position. However, $\mathrm{P}_{\mathrm{ES}}$ closely reflected the $\mathrm{P}_{\mathrm{PL}}$ of regions adjacent to the esophagus (dependent to middle lung). In our study, we could not control for the chest-tube position. The chest-tubes had multiple holes, theoretically limiting the magnitude of the problem, and providing an intermediate value. But the patency of the different holes could not be ascertained, and this may explain part of the discrepancy observed. Occlusion of the drain by secretions can certainly impact the measurements. We tried to avoid this problem by flushing some air prior to the measurement, although it is still possible that residual secretions interfered with the column of air. As illustrated in the figure 1 , the pleural signals were frequently attenuated in our cases. The adequate transmission of the pressure variation within the breathing cycle is an important criterion for the accuracy of the pressure measurement [33]. It is probable that the pressure transmission was not excellent over time with our method. However, we consider that the equilibrium obtained after the little injection of air likely reflected the pressure in the pleural space at least for this brief recording time. Due to the attenuation of the intra-breath pressure changes, we could not reliably assess the relationship between the different pressures at end inspiration. The presence of pleural effusion can also alter $\mathrm{P}_{\mathrm{PL}}$ recordings, but this was excluded in our cases.

The methods used to measure the esophageal pressure need also to be discussed. As opposed to the esophageal balloon technique, the use of a catheter-mounted miniature pressure transducer like the Gaeltec probe allows to eliminate the effect of the esophageal wall and the impact of the mechanical properties of the balloon on measurements, as well as the influence of the volume of air injected in the balloon. However, the measurement of absolute values of $\mathrm{P}_{\mathrm{ES}}$ has been previously questioned by Stell et al. [34] and Beda et al. [35]. In addition, in our study, the protocol to check the position of the Gaeltec probe was limited by the clinical context (no airway occlusion nor chest compression). Since the Baydur method [17] was impossible to perform, the position of the catheter was estimated by the nose to ear to xiphisternum distance and verified by the presence of cardiac oscillation on the $\mathrm{P}_{\mathrm{ES}}$ trace. However, we consider that the impact of such limitation is small as compared to the great difference we observed between $\mathrm{P}_{\mathrm{CH}-\mathrm{TUBE}}$ and $\mathrm{P}_{\mathrm{ES}}$.

The alternative method to measure $\mathrm{P}_{\mathrm{ES}}$ using the feeding tube was tested in an attempt to obtain a very simple method, based on material already in place in most patients, and easy to implement in any condition. Previous data in adult patients have shown that a fluid filled tube could be relatively accurate to estimate $\mathrm{P}_{\mathrm{ES}}$ [36]. We rather tested an air-filled catheter for two reasons. First, we expected that the signal would be attenuated after a few minutes, but our goal was to obtain a brief estimation of the $\mathrm{P}_{\mathrm{ES}}$ (rapidly after the air flush), not a continuous monitoring. Second, we thought that air flush would be more easily accepted by the parents and the treating teams. Posteriori, the attenuation of the pressure fluctuations rapidly after the flush makes it difficult to ascertain the adequate pressure transmission. The alteration of the feeding tube patency by gastric secretions, or by feeding formula or enteral medications may also have plaid a role. Further studies should look at the accuracy of fluid-filled feeding tube.

Other limits of our study include the single-center design, the small sample size, and the heterogeneity of the patients. Importantly, most children were younger than one year of age, and it is uncertain if our findings are generalizable to older children. End-inspiratory and end-expiratory pauses have not been applied, precluding the complete elimination of the resistance influence at zero flow.

The methods that we used in this study to record esophageal pressure had clear limitations and we may wonder if balloon catheters would have provided better results. We did not use balloon catheters in this study, because we had an important experience with the Gaeltec probe and because of the major sensitivity of the esophageal balloon filling on the pressure measurements. However, filling titration methods have been described to overcome this problem [37,38]. The optimal filling volume can be determined as the volume which provides the most accurate esophageal pressure swing [33]. In a bench model, Hotz et al. also described a plateau in the relationship between balloon inflation and $\mathrm{P}_{\mathrm{ES}}$ measurements [38]. Around this optimal filling volume (0.2-0.6 ml), the catheter accurately reflects the $\mathrm{P}_{\mathrm{ES}}$ in the bench model. This promising approach could solve several problems that we encountered, but it should be validated in a clinical context. In particular, small imprecision in inflation could probably link to clinically significant errors in esophageal pressure estimation.

\section{Conclusion}

The recent international consensus conference on pediatric acute respiratory distress syndrome PALICC [9] stresses that $\mathrm{P}_{\mathrm{ES}}$ measurement could be useful, mostly based on data extrapolated from adults, although more research and validation in this field were advocated. Our results suggest that prior to consider its use in clinical practice, in particular for the titration of the ventilatory support, it is essential to conduct more research in order to validate the measurement technique of $\mathrm{P}_{\mathrm{ES}}$ and confirm that it can accurately reflect the $\mathrm{P}_{\mathrm{PL}}$. This is far from what we observed in our series. Awaiting those future studies, we argue for prudence, and suggest not using $\mathrm{P}_{\mathrm{ES}}$ for the titration of ventilation support in children outside a research context.

\section{Conflict of interest}

GM, NN, NP and SE have no conflict of interest to declare. PJ is supported by a scholarship award of the Fonds de Recherche du Québec-Santé, Ministry of Health and Sainte-Justine Hospital. PJ was a consultant for Sage Therapeutic inc, was invited to a congress by Medunik Inc and Covidien. GE's research program is supported by a scholarship award by the Fonds de Recherche du Québec - Santé. $\mathrm{He}$ is currently leading a feasibility study in neonatal ventilation which is financially supported by Maquet Critical Care. The research of BF is supported by the Association Française contre les Myopathies (AFM), Assistance Publique-Hôpitaux de Paris, Inserm, Université Paris Descartes, ADEP Assistance, ASV Santé, S2A Santé and IP Santé Domicile.

\section{Author's contribution}

GM, NP, PJ, BF and GE designed the study. GM, NN, SE and GE obtained and analyzed the data from Esophageal and Pleural Pressures. 
GM and NN wrote the manuscript, which was reviewed, edited, and approved by all authors.

As the corresponding author, GM has full access to all the data in the study and has final responsibility for the decision to submit for publication.

\section{References}

1. Akoumianaki E, Maggiore SM, Valenza F, Bellani G, Jubran A, et al. (2014) The application of esophageal pressure measurement in patients with respiratory failure. Am J Respir Crit Care Med 189: 520-531. [Crossref]

2. JH B (1949) Intraesophageal pressure and lung elasticity. University of Groningen.

3. Higgs BD, Behrakis PK, Bevan DR, Milic-Emili J (1983) Measurement of pleural pressure with esophageal balloon in anesthetized humans. Anesthesiology 59: 340-343. [Crossref]

4. Hurewitz AN, Sidhu U, Bergofsky EH, Chanana AD (1984) How alterations in pleural pressure influence esophageal pressure. J Appl Physiol Respir Environ Exerc Physiol 56: 1162-1169. [Crossref]

5. Cherniack RM, Farhi LE, Armstrong BW, Proctor DF (1955) A comparison of esophageal and intrapleural pressure in man. J Appl Physiol 8: 203-211. [Crossref]

6. Thille AW, Cabello B, Galia F, Lyazidi A, Brochard L (2008) Reduction of patientventilator asynchrony by reducing tidal volume during pressure-support ventilation. Intensive Care Med 34: 1477-1486. [Crossref]

7. Talmor D, Sarge T, Malhotra A, O'Donnell CR, Ritz R, et al. (2008) Mechanical ventilation guided by esophageal pressure in acute lung injury. $N$ Engl J Med 359: 2095-2104. [Crossref]

8. Jubran A, Grant BJ, Laghi F, Parthasarathy S, Tobin MJ (2005) Weaning prediction: esophageal pressure monitoring complements readiness testing. Am J Respir Crit Care Med 171: 1252-1259. [Crossref]

9. The Pediatric Acute Lung Injury Consensus Conference Group (2015) Pediatric acute respiratory distress syndrome: consensus recommendations from the Pediatric Acute Lung Injury Consensus Conference. Pediatr Crit Care Med 16: 428-439. [Crossref]

10. Owens RL, Stigler WS, Hess DR (2008) Do newer monitors of exhaled gases, mechanics, and esophageal pressure add value? Clin Chest Med 29: 297-312, vi-vii. [Crossref]

11. Drummond GB, Wright AD (1983) Inaccuracy of oesophageal pressure for pleural pressure estimation in supine anaesthetized subjects. Br J Anaesth 55: 585-593. [Crossref]

12. Washko GR, O'Donnell CR, Loring SH (1985) Volume-related and volumeindependent effects of posture on esophageal and transpulmonary pressures in healthy subjects. J Appl Physiol 100: 753-758. [Crossref]

13. Van de Woestijne KP, Trop D, Clément J (1971) Influence of the mediastinum on the measurement of esophageal pressure and lung compliance in man. Pflugers Arch 323: 323-341. [Crossref]

14. Talmor D, Sarge T, O’Donnell CR, Ritz R, Malhotra A, et al. (2006) Esophageal and transpulmonary pressures in acute respiratory failure. Crit Care Med 34: 1389-1394. [Crossref]

15. Brander L, Ranieri VM, Slutsky AS (2006) Esophageal and transpulmonary pressure help optimize mechanical ventilation in patients with acute lung injury. Crit Care Med 34: 1556-1558. [Crossref]

16. Loring SH, O'Donnell CR, Behazin N, Malhotra A, Sarge T, et al. (2010) Esophagea pressures in acute lung injury: do they represent artifact or useful information about transpulmonary pressure, chest wall mechanics, and lung stress? J Appl Physiol 108: 515-522. [Crossref]

17. Baydur A, Behrakis PK, Zin WA, Jaeger M, Milic-Emili J (1982) A simple method for assessing the validity of the esophageal balloon technique. Am Rev Respir Dis 126: 788-791. [Crossref]
18. Koo TK, Li MY (2016) A Guideline of Selecting and Reporting Intraclass Correlation Coefficients for Reliability Research. J Chiropr Med 15: 155-163. [Crossref]

19. Bland JM, Altman DG (2003) Applying the right statistics: analyses of measurement studies. Ultrasound Obstet Gynecol 22: 85-93. [Crossref]

20. Milic-Emili J, Mead J, Turner JM, Glauser EM (1964) Improved technique for estimating pleural pressure from esophageal balloons. J Appl Physiol 19: 207-211. [Crossref]

21. Buytendijk HJ (1949) Electriche Drukkerij I. Groningen: Oppenheim N.V.

22. Khemani RG, Hotz J, Morzov R, Flink RC, Kamerkar A, et al. (2016) Pediatric extubation readiness tests should not use pressure support. Intensive Care Med 42: 1214-1222. [Crossref]

23. Mortamet G, Khirani S, Amaddeo A, Emeriaud G, Renolleau S, et al. (2017) Esogastric pressure measurement to assist noninvasive ventilation indication and settings in infants with hypercapnic respiratory failure: A pilot study. Pediatr Pulmonol 52: 1187 1193. [Crossref]

24. Dinwiddie R, Russell G (1972) Relationship of intraesophageal pressure to intrapleural pressure in the newborn. J Appl Physiol 33: 415-417. [Crossref]

25. Mead J, Gaensler EA (1959) Esophageal and pleural pressures in man, upright and supine. J Appl Physiol 14: 81-83. [Crossref]

26. Guerin C, Richard JC (2012) Comparison of 2 correction methods for absolute values of esophageal pressure in subjects with acute hypoxemic respiratory failure, mechanically ventilated in the ICU. Respir Care 57: 2045-2051. [Crossref]

27. Grasso S, Terragni P, Birocco A, Urbino R, Del Sorbo L, et al. (2012) ECMO criteria for influenza A (H1N1)-associated ARDS: role of transpulmonary pressure. Intensive Care Med 38: 395-403. [Crossref]

28. Chiumello D, Carlesso E, Cadringher P, Caironi P, Valenza F, et al. (2008) Lung stress and strain during mechanical ventilation for acute respiratory distress syndrome. Am J Respir Crit Care Med 178: 346-355. [Crossref]

29. Hustead R, Avery M (1964) The lung and its disorders in the newborn infant: Philadelphia: Saunders.

30. Feller-Kopman D, Parker MJ, Schwartzstein RM (2009) Assessment of pleural pressure in the evaluation of pleural effusions. Chest 135: 201-209. [Crossref]

31. Salamonsen M, Ware R, Fielding D (2014) A new method for performing continuous manometry during pleural effusion drainage. Respiration 88: 61-66. [Crossref]

32. Yoshida T, Amato MBP, Grieco DL, Chen L, Lima CAS, et al. (2018) Esophageal Manometry and Regional Transpulmonary Pressure in Lung Injury. Am J Respir Crit Care Med 197: 1018-1026. [Crossref]

33. Mojoli F, Iotti GA, Torriglia F, Pozzi M, Volta CA, et al. (2016) In vivo calibration of esophageal pressure in the mechanically ventilated patient makes measurements reliable. Crit Care 20: 98. [Crossref]

34. Stell IM, Tompkins S, Lovell AT, Goldstone JC, Moxham J (1999) An in vivo comparison of a catheter mounted pressure transducer system with conventional balloon catheters. Eur Respir J 13: 1158-1163. [Crossref]

35. Beda A, Guldner A, Carvalho AR, Zin WA, Carvalho NC, et al. (2014) Liquid- and air-filled catheters without balloon as an alternative to the air-filled balloon catheter for measurement of esophageal pressure. PLoS One 9: e103057. [Crossref]

36. Verscheure S, Massion PB, Gottfried S, Goldberg P, Samy L, et al. (2016) Measurement of pleural pressure swings with a fluid-filled esophageal catheter vs pulmonary artery occlusion pressure. J Crit Care 37: 65-71. [Crossref]

37. Mauri T, Yoshida T, Bellani G, Goligher EC, Carteaux G, et al. (2016) Esophageal and transpulmonary pressure in the clinical setting: meaning, usefulness and perspectives. Intensive Care Med 42: 1360-1373. [Crossref]

38. Hotz JC, Sodetani CT, Van Steenbergen J, Khemani RG, Deakers TW, et al. (2018) Measurements obtained from esophageal balloon catheters are affected by the esophageal balloon filling volume in children with ARDS. Respir Care 63: 177-186. [Crossref]

Copyright: (C2018 Mortamet G. This is an open-access article distributed under the terms of the Creative Commons Attribution License, which permits unrestricted use, distribution, and reproduction in any medium, provided the original author and source are credited. 